\title{
Association between MMP-2 expression and prostate cancer: A meta-analysis
}

\author{
TIANCHENG XIE*, BINBIN DONG* , YANGYE YAN*, GUANGHUI HU and YUNFEI XU
}

Department of Urology, Shanghai Tenth People's Hospital, Tongji University School of Medicine, Shanghai 200072, P.R. China

Received August 31, 2015; Accepted November 27, 2015

DOI: 10.3892/br.2015.553

\begin{abstract}
Matrix metalloproteinase-2 (MMP-2) is a member of the MMP family, which is associated with numerous types of cancer. Although it has been widely reported, the prognostic value of MMP-2 expression in prostate cancer ( $\mathrm{PCa}$ ) remains controversial. Thus, the present meta-analysis was conducted to investigate the association and prognostic value of MMP-2 expression in PCa. PubMed, Cochrane Library and the China National Knowledge Infrastructure databases were searched for all the published case-control studies on the association between MMP-2 expression and PCa until July 2015. The odd ratios (ORs) and 95\% confidence intervals (CIs) were used to estimate the association of MMP-2 expression and PCa. ORs and 95\% CIs were applied to clarify this association. Several subgroup analyses were also conducted according to different indexes in the case group. In total, 8 studies including 675 patients were included in the final meta-analysis. The results of the meta-analysis showed that MMP-2 expression in the PCa group was significantly higher than that in the benign prostatic hyperplasia $(\mathrm{BPH})$ group (95\% CI, 0.06-0.15; $\mathrm{Z}=10.48$; $\mathrm{P}<0.00001)$. Furthermore, MMP-2 expression was significantly associated with Gleason Score (95\% CI, 0.18-0.68; $\mathrm{Z}=3.09 ; \mathrm{P}=0.002)$ and clinical stages $(95 \% \mathrm{CI}$, $0.12-0.82 ; \mathrm{Z}=2.36 ; \mathrm{P}=0.02)$, and not significantly associated with Gleason score serum prostate specific antigen $(95 \% \mathrm{CI}$, $0.30-1.66 ; \mathrm{Z}=0.80 ; \mathrm{P}=0.43$ ). In conclusion, MMP-2 is overexpressed in $\mathrm{PCa}$ tissues compared with $\mathrm{BPH}$. The expression of MMP-2 was significantly associated with the grade of PCa malignancy.
\end{abstract}

Correspondence to: Dr Yunfei Xu, Department of Urology, Shanghai Tenth People's Hospital, Tongji University School of Medicine, 301 Middle Yanchang Road, Shanghai 200072, P.R. China E-mail: xuyunfeibb@sina.com

*Contributed equally

Key words: matrix metalloproteinase-2, prostate cancer, benign prostatic hyperplasia, meta-analysis

\section{Introduction}

Prostate cancer $(\mathrm{PCa})$ is one of the most commonly diagnosed malignant tumors and the second cause of cancer in males $(1,2)$. Due to the changes of population ages and diet structure, the global incidence of PCa has increased annually. The pathogenesis of PCa remains to be fully elucidated. Therefore, identifying a marker with a high correlation with occurrence and development of $\mathrm{PCa}$ is important for early diagnosis and treatment of $\mathrm{PCa}$.

Matrix metalloproteinases (MMPs) are a series of protein hydrolases, which are closely associated with tumor growth, invasion and metastasis $(3,4)$. MMPs can degrade the extracellular matrix (ECM), and can control the formation of tumor blood vessels (5). They have numerous subtypes, and MMP-2 is one of the most researched. There are a number of studies regarding the association between MMP-2 and PCa, which showed that the serum MMP-2 level was significantly higher compared to the control subjects (6-9). However, the impact of MMP-2 expression on the progress of PCa patients remains disputed. Certain studies have shown that MMP-2 has a high expression level in $\mathrm{PCa}$; however, the sample sizes of these studies were small, or they were not contrasted further to the case group. Thus, the present meta-analysis was performed to explore the association of the level of MMP-2 expression and PCa.

\section{Materials and methods}

Search strategy. The following electronic databases were comprehensively searched: PubMed, Cochrane Library and China National Knowledge Infrastructure performed until July 2015. The following search terms were used: 'MMP-2' or 'matrix metalloproteinase-2', 'prostate cancer' or 'prostate tumor' or 'prostate'. Subsequently, the literature was retrieved for further screening.

Study selection. The following criteria was used to evaluate the retrieval literature, which is consistent with the analysis included in the request: It should be the original and independent research; it must be the malignant tumor originating in the prostate; the association between MMP-2 expression and PCa should be shown; it must be a case-control study, with benign prostatic hyperplasia (BPH) as the control; the MMP-2 expression should be detected in formalin-fixed and 
paraffin-embedded (FFPE) tumor tissues. Simultaneously, the following exclusion criteria was used: Cell lines or animals were used; review articles; and the data was incomplete.

Data extraction. Data were extracted from the included studies as follows: Surname of the first author, the year of publishing, country, median age of patients, study sample size, the percentage of MMP-2 positive, survival outcomes, method of hazard ratio (HR) estimation, method of survival analysis, HR and 95\% confidence interval (CI), and odds ratio (OR). Two investigators (Tiancheng Xie and Binbin Dong) extracted the data independently. Any disagreement regarding data was resolved by another investigator (Yangye Yan) to adjudicate the result.

Study quality. Two independent authors (Tiancheng Xie and Binbin Dong) evaluated the quality of the included studies in the meta-analysis, according to the Newcastle-Ottawa Scale (NOS) for case-control studies. The NOS is from 0 to 9 stars. Any controversy was solved by discussion with the third investigator (Yangye Yan) to adjudicate any disagreement.

Statistical analysis. Heterogeneity was analyzed by calculating the $\mathrm{Q}$ test statistics. When the data suggest $\mathrm{P}>0.10$ with no significant heterogeneity, the fixed effects model was used, otherwise, the random effects model was employed. Due to the rare incidence rate of $\mathrm{PCa}, \mathrm{HR}$ could be approximately equal to OR. Therefore, OR was used instead of HR. Egger's funnel plot was explored to identify if there was any evidence of publication bias (10).

\section{Results}

Information from the literature. Based on the above search strategy, 395 relevant studies were identified. Subsequently, the initial screening occurred by reading the title and abstract of these retrieved studies. A total of 355 studies were excluded as they did not focus on the association of MMP-2 expression and PCa. Following this, 1 study was excluded as it was not associated to MMP-2 or PCa. Following reading of the remaining studies, 29 were excluded as they were continuous variables or others form of data, or non-comprehensive data. In addition, 2 studies were excluded that were not case-control studies. Finally, 8 case-control studies (2 in English and 6 in Chinese) were included in the meta-analysis $(9,11-17)$. The selection process is shown in Fig. 1.

Study characteristics. All the studies were published between 2005 and 2014. There were 7 studies of the Asian population $(9,11-13,15-17)$ and 1 of the Caucasian population (14). A total of 7 studies compared the positive rate of MMP-2 between the PCa and BPH groups, and 5 of these compared the MMP-2 positive rate subdivided by the Gleason score in the cancer group $(11,12,15-17)$.

Furthermore, in the PCa group, 2 studies compared the positive rate of MMP-2 between low and high serum prostate-specific antigen (PSA) groups $(12,15)$, and 2 studies compared the positive rate of MMP-2 between clinical stages (Jewett stages) AB and CD groups $(11,16)$. An average NOS

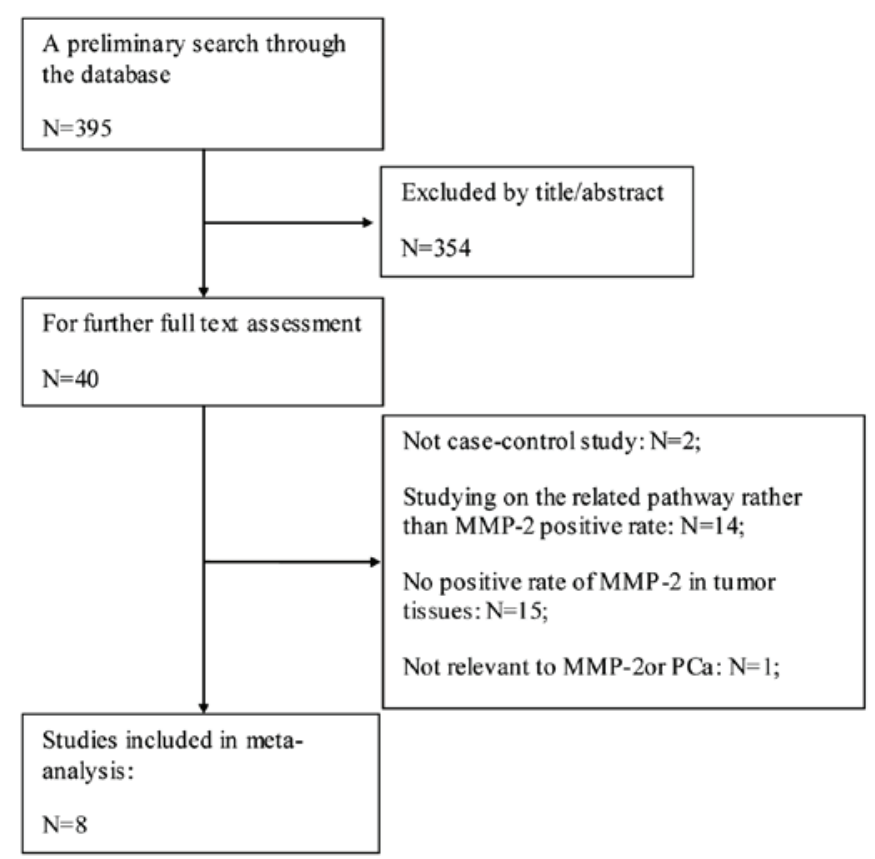

Figure 1. Flowchart presenting the steps of the literature search and selection. MMP-2, matrix metalloproteinase-2.

score of 6 indicated a reliable quality. The characteristics of the included studies are shown in Table I.

\section{Meta-analysis}

BPH and cancer. There were 8 studies with a total of 498 cases and 177 controls that compared the differences of the MMP-2 positive rate between case-control groups. There was no significant heterogeneity $\left(\mathrm{I}^{2}=0 \%, \mathrm{P}=0.55\right)$, and the pooled $\mathrm{OR}$ was 0.09 (95\% CI, 0.06-0.15; $\mathrm{Z}=10.58 ; \mathrm{P}<0.00001)$. The results showed that the MMP-2 expression was significantly associated with PCa (Fig. 2).

Gleason score. There were 5 studies that compared the differences in the positive rate of MMP-2 in the Gleason score high and low groups. The combined OR was 0.35 (95\% CI, $0.18-0.68 ; \mathrm{Z}=3.09 ; \mathrm{P}=0.002)$ with no significant heterogeneity $\left(\mathrm{I}^{2}=7 \%, \mathrm{P}=0.37\right)$. Therefore, the expression level of MMP-2 has a positive association with the Gleason score of patients (Fig. 3).

Subgroup analysis. In addition, there were 2 subgroup analyses. One was regarding serum PSA, and it explored whether MMP-2 has a correlation between high and low serum PSA. The heterogeneity was not significant $\left(\mathrm{I}^{2}=56 \%\right.$, $\mathrm{P}=0.13$ ), and the pooled OR was 0.71 (95\% CI, 0.30-1.66; $\mathrm{Z}=0.80 ; \mathrm{P}=0.43)$. Another compared the positive rate of MMP-2 between clinical stages $\mathrm{AB}$ and $\mathrm{CD}$. The pooled $\mathrm{OR}$ was 0.32 (95\% CI, 0.12-0.82; $\mathrm{Z}=2.36 ; \mathrm{P}=0.02$ ) without significant heterogeneity $\left(I^{2}=29 \%, P=0.23\right)$. These results suggested that MMP-2 was significantly correlated with clinical stages, but not significantly correlated with serum PSA (Fig. 4).

Publication bias and sensitivity analysis. In order to test whether the final result of this meta-analysis was affected by individual study and gauge the stability of the results, a sensitivity analysis was conducted (Fig. 5). The pooled OR in the meta-analysis was not effect by single study. The result of 
Table I. Characteristics of the eligible studies in the meta-analysis.

\begin{tabular}{|c|c|c|c|c|c|c|c|c|c|c|c|c|c|}
\hline \multirow[b]{3}{*}{ First author, year } & \multirow{3}{*}{ Ethnicity } & \multirow{3}{*}{$\begin{array}{c}\text { Source } \\
\text { of controls }\end{array}$} & \multirow{2}{*}{\multicolumn{2}{|c|}{ Sample size, $\mathrm{n}$}} & \multirow{2}{*}{\multicolumn{2}{|c|}{ Age, years }} & \multirow{2}{*}{\multicolumn{2}{|c|}{$\begin{array}{l}\text { Gleason score } \\
\text { (in case) }\end{array}$}} & \multicolumn{4}{|c|}{ Subgroups, n } & \multirow[b]{3}{*}{ (Refs.) } \\
\hline & & & & & & & & & \multicolumn{2}{|c|}{ PSA } & \multicolumn{2}{|c|}{ Clinical stages } & \\
\hline & & & Case & Control & Case & Control & Low $(\leq 7)$ & High $(>7)$ & Low & High & $\mathrm{AB}$ & $\mathrm{CD}$ & \\
\hline Ma, 2014 & Asian & $\mathrm{HB}$ & 30 & 60 & 67 & 65 & 24 & 36 & 25 & 35 & $\mathrm{~N}$ & $\mathrm{~N}$ & (12) \\
\hline Li, 2013 & Asian & $\mathrm{HB}$ & 20 & 78 & $\mathrm{~N}$ & $\mathrm{~N}$ & $\mathrm{~N}$ & $\mathrm{~N}$ & $\mathrm{~N}$ & $\mathrm{~N}$ & $\mathrm{~N}$ & $\mathrm{~N}$ & (13) \\
\hline Escaff, 2011 & Caucasian & HB & 50 & 133 & $54-70$ & $44-79$ & $\mathrm{~N}$ & $\mathrm{~N}$ & $\mathrm{~N}$ & $\mathrm{~N}$ & $\mathrm{~N}$ & $\mathrm{~N}$ & (14) \\
\hline Jia, 2010 & Asian & HB & 20 & 40 & 59 & 81 & 26 & 14 & $\mathrm{~N}$ & $\mathrm{~N}$ & 15 & 25 & (11) \\
\hline Wu, 2009 & Asian & $\mathrm{HB}$ & 20 & 48 & $\mathrm{~N}$ & $\mathrm{~N}$ & 33 & 15 & $\mathrm{~N}$ & $\mathrm{~N}$ & $\mathrm{~N}$ & $\mathrm{~N}$ & (17) \\
\hline Zhong, 2008 & Asian & $\mathrm{HB}$ & 62 & 15 & $73.9 \pm 12.1$ & $\mathrm{~N}$ & $\mathrm{~N}$ & $\mathrm{~N}$ & $\mathrm{~N}$ & $\mathrm{~N}$ & $\mathrm{~N}$ & $\mathrm{~N}$ & (9) \\
\hline Wu, 2005 & Asian & HB & 12 & 46 & $<40$ & $53-70$ & 39 & 7 & $\mathrm{~N}$ & $\mathrm{~N}$ & 26 & 20 & (16) \\
\hline Zhang, 2005 & Asian & $\mathrm{HB}$ & 10 & 51 & 34 (17) & $31(20)$ & 34 & 17 & 31 & 20 & $\mathrm{~N}$ & $\mathrm{~N}$ & (15) \\
\hline
\end{tabular}

PSA, prostate-specific antigen; HB, hospital-based; N, none; $\mathrm{AB}$ and $\mathrm{CD}, 4$ clinical stages (Jewett stages).

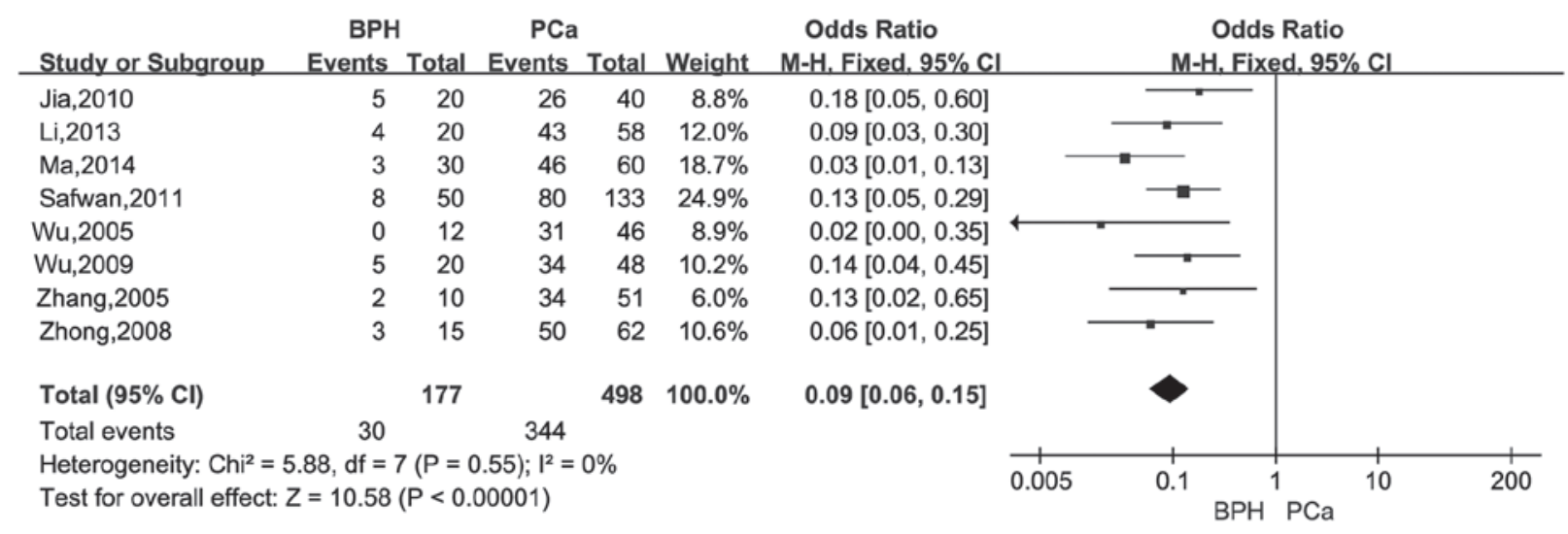

Figure 2. Significant association of matrix metalloproteinase-2 expression with prostate cancer (PCa). BPH, benign prostatic hyperplasia; CI, confidence interval.

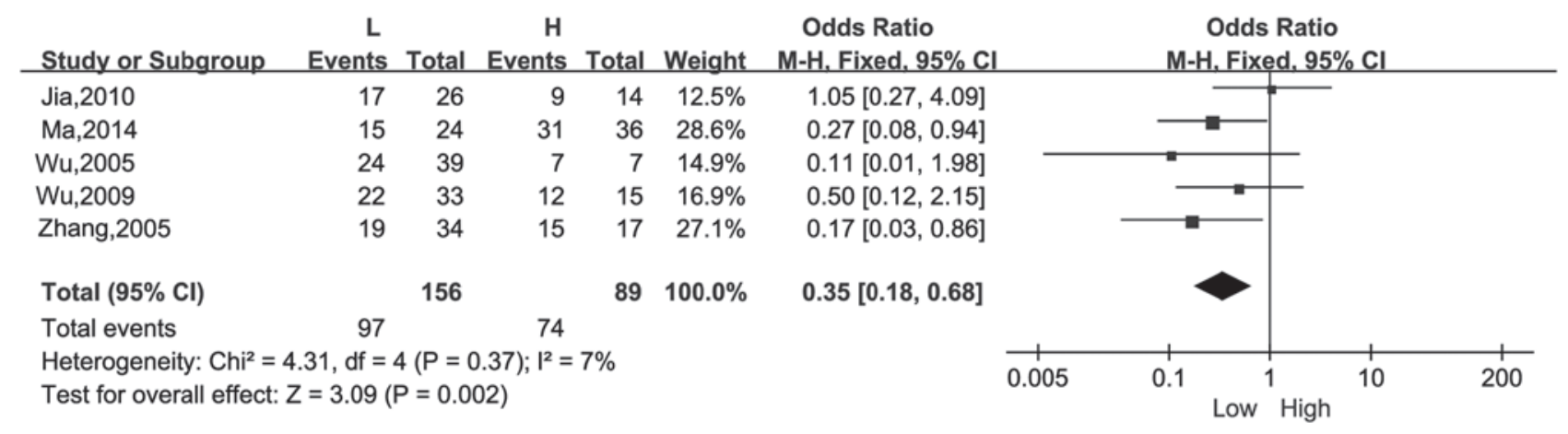

Figure 3. Significant association of matrix metalloproteinase-2 expression with the Gleason score of patients. CI, confidence interval.

Egger's regression test showed the asymmetrical distribution in the funnel plot in the positive rate of MMP-2 between high and low Gleason score groups (Egger's test, $\mathrm{t}=-2.00$ ).

\section{Discussion}

Worldwide, the incidence of PCa ranked second in all male malignant tumors (18). In the USA, the incidence of PCa has been higher compared to lung cancer, and it became the first tumor hazard in male health. In Asia, the incidence of $\mathrm{PCa}$ is much lower compared to Europe, America and other developed countries. However, with the development of the economy and technology, and changes to life and eating habits, the incidence of $\mathrm{PCa}$ is in a growing trend in recent years, and the growth rate is higher than the developed countries in Europe and America. 


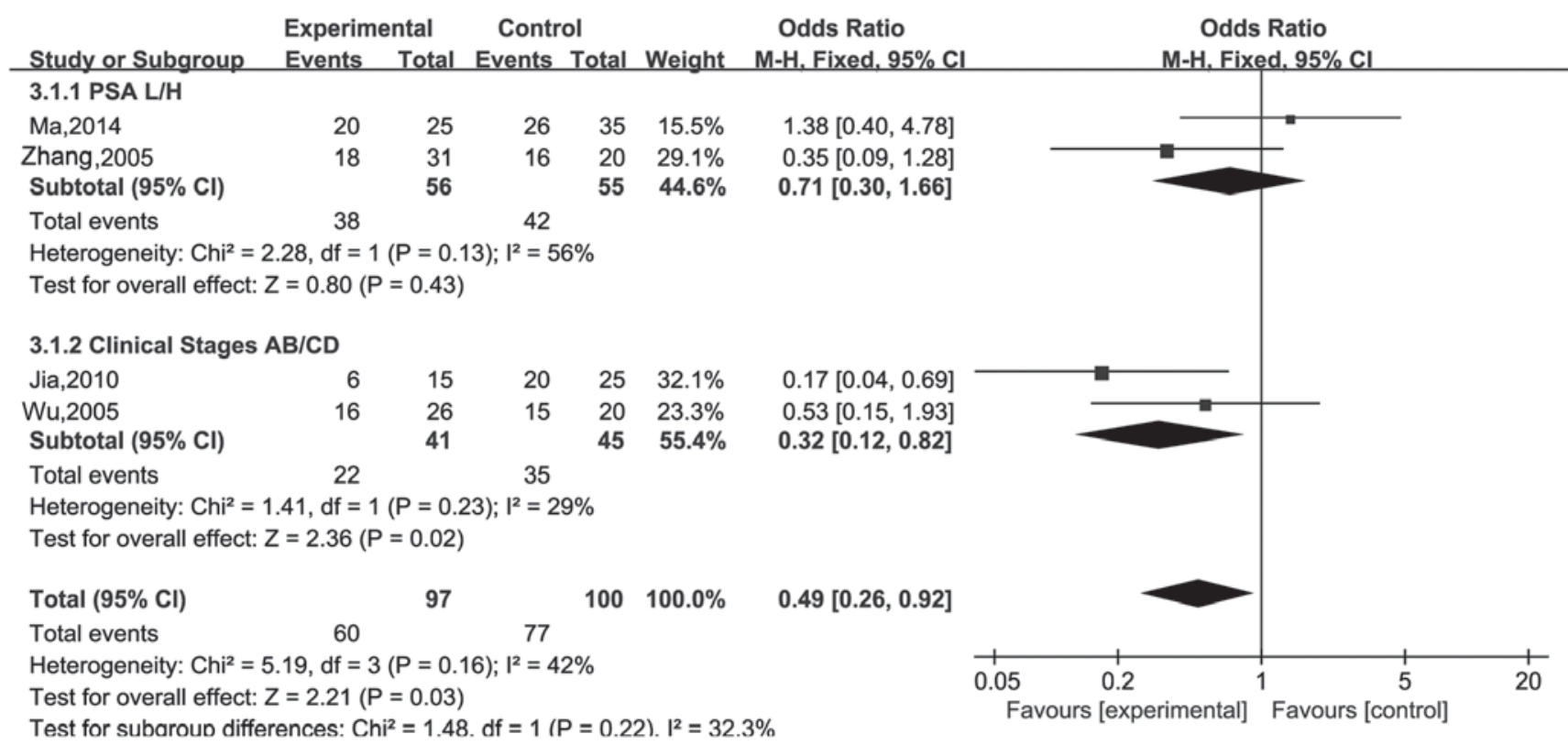

Figure 4. Significant association of matrix metalloproteinase-2 expression with clinical stages, but no significant correlation with serum prostate-specific antigen (PSA). CI, confidence interval.

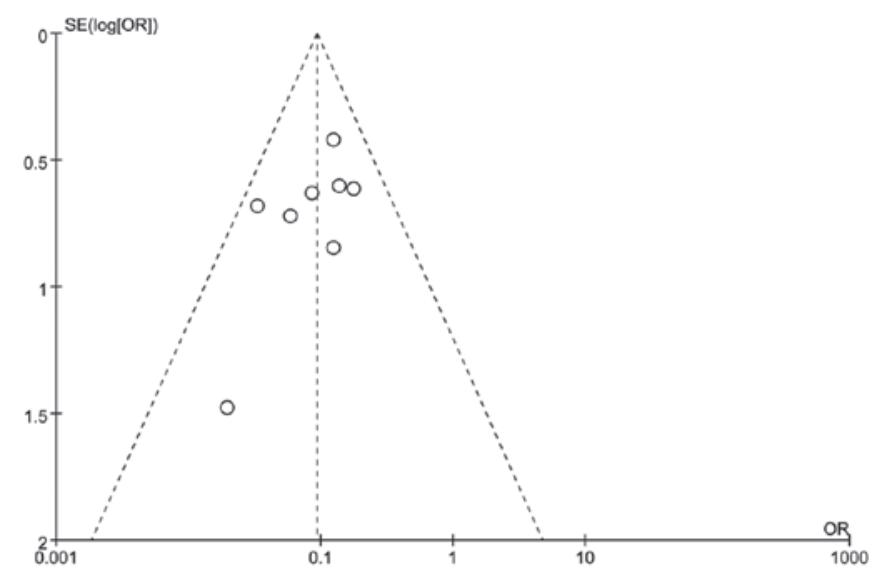

Figure 5. Funnel plot of publication bias on the differences of matrix metalloproteinase-2. Expression between benign prostatic hyperplasia and prostate cancer patients. OR, odds ratio.

Early PCa often has no symptoms, and it is always diagnosed in the advanced stage following the appearance of symptoms (19). It will lose some selectivity for treatment, and the prognosis is poor. Therefore, how to early diagnose of $\mathrm{PCa}$ and monitor the prognosis of $\mathrm{PCa}$ is extremely important.

Clinical tumor-node-metastasis (TNM) staging of PCa and the malignant degree, Gleason score of $\mathrm{PCa}$ risk grade, and the prognosis have an important reference value. In PCa TNM staging, lymph node metastasis of judgment depends mainly on the computed tomography (CT) scanning, magnetic resonance imaging (MRI) or biopsy. Open or laparoscopic lymph node dissection is the gold standard for $\mathrm{N}$ staging. However, the specificity of CT, MRI and lymph node biopsy are invasive examinations, which certain patients find it difficult to accept. Currently, PSA is widely applied in the clinic, and it has an important role in the diagnosis of $\mathrm{PCa}$; however, when the
PSA value is in the $4-10 \mathrm{ng} / \mathrm{ml}$ gray zone, it will reduce the sensitivity and specificity of PSA.

MMPs are ECM-degrading enzymes belonging to a family of zinc- and calcium-dependent endopeptidases. They are important for cancer invasion and metastasis (20) and they have a significant role for ECM degradation $(21,22)$. MMP-2 is one of the MMP families. It has a critical role in tumor growth and metastasis (23-25). There are studies that have identified that the MMP-2 content in the serum is associated with the grading and malignant degree of PCa $(26,27)$. Certain studies observed that MMP-2 may have the potential to be used as a molecular marker for PCa (7). MMP-2 may be used as a predictor of PCa (8). In addition, certain studies showed an increased or decreased expression level of MMP-2 through the regulation of an associated MMP-2 pathway, and tumor cell invasion could be promoted or inhibited in PCa cells (28).

Recently, the association between MMP-2 expression and PCa has been investigated by certain studies. However, there remain certain disputes regarding the result, and the sample sizes are small. Additionally, there is no meta-analysis reporting the association of MMP-2 expression and $\mathrm{PCa}$. Therefore, the present meta-analysis was conducted to explore the association between MMP-2 expression and PCa patients.

There are a total of 8 studies, including 498 cases and 177 controls, in this meta-analysis. Between the BPH and PCa groups, there was no significant heterogeneity $\left(\mathrm{I}^{2}=0 \%\right.$, $\mathrm{P}=0.55)$, and the pooled OR was 0.09 (95\% CI, 0.06-0.15; $\mathrm{Z}=10.58$; $\mathrm{P}<0.00001)$, indicating that MMP-2 overexpression was associated with PCa. The pooled OR with its 95\% CI indicated that the expression of MMP-2 increased with the increase of the PCa Gleason score. Subgroup analysis showed that the expression of MMP-2 was positively correlated with clinical stage in PCa patients. Although the expression of MMP-2 was not significantly correlated with serum PSA, MMP-2 may be a potential marker in $\mathrm{PCa}$ patients. It may be used in early diagnosis and the evaluation of prognosis regarding $\mathrm{PCa}$. 
Although a comprehensive search was performed, along with rigorous statistical data analysis, certain limitations remain. Firstly, the literature that was searched may predominantly accept their positive results, leading to expansion of the results of the meta-analysis. Secondly, certain studies were not included in the meta-analysis due to the non-uniform data standards. Thirdly, only studies written in Chinese or English were included in the meta-analysis even though there was no language restriction. Fourthly, the majority of the included studies are based on the population of Asian countries.

In conclusion, taken together the present meta-analysis showed that MMP-2 was highly expressed in PCa patients compared with BPH patients. The expression of MMP-2 was closely correlated with Gleason score and clinical stages in PCa patients. Therefore, MMP-2 can serve as an indicator in PCa patients. However, there are also certain limitations. The additional studies should explore the prognostic value of serum MMP-2 in PCa patients and clarify the prognostic significance of MMP-2 expression in PCa patients.

\section{Acknowledgements}

The present study was funded by the National Natural Science Foundation of China (grant no. 81370699). The authors acknowledge the reviewers for their comments on this study.

\section{References}

1. Ferlay J, Shin HR, Bray F, Forman D, Mathers C and Parkin DM: Estimates of worldwide burden of cancer in 2008: GLOBOCAN 2008. Int J Cancer 127: 2893-2917, 2010.

2. Siegel RL, Miller KD and Jemal A: Cancer statistics, 2015. CA Cancer J Clin 65: 5-29, 2015.

3. Nemeth JA, Yousif R, Herzog M, Che M, Upadhyay J, Shekarriz B, Bhagat S, Mullins C, Fridman R and Cher ML: Matrix metalloproteinase activity, bone matrix turnover, and tumor cell proliferation in prostate cancer bone metastasis. J Natl Cancer Inst 94: 17-25, 2002.

4. Lokeshwar BL: MMP inhibition in prostate cancer. Ann NY Acad Sci 878: 271-289, 1999.

5. Song H, Li Y, Lee J, Schwartz AL and Bu G: Low-density lipoprotein receptor-related protein 1 promotes cancer cell migration and invasion by inducing the expression of matrix metalloproteinases 2 and 9. Cancer Res 69: 879-886, 2009.

6. Srivastava P, Lone TA, Kapoor R and Mittal RD: Association of promoter polymorphisms in MMP2 and TIMP2 with prostate cancer susceptibility in North India. Arch Med Res 43: 117-124, 2012.

7. dos Reis ST, Villanova FE, Andrade PM, Pontes J Jr, de Sousa-Canavez JM, Sañudo A, Antunes AA, Dall'oglio MF, Srougi M and Moreira Leite KR: Matrix metalloproteinase-2 polymorphism is associated with prognosis in prostate cancer Urol Oncol 28: 624-627, 2010.

8. TrudelD, Fradet Y,Meyer F, HarelF and Têtu B: Membrane-type-1 matrix metalloproteinase, matrix metalloproteinase 2 , and tissue inhibitor of matrix proteinase 2 in prostate cancer: Identification of patients with poor prognosis by immunohistochemistry. Hum Pathol 39: 731-739, 2008.
9. Zhong WD, Han ZD, He HC, Bi XC, Dai QS, Zhu G, Ye YK, Liang YX, Qin WJ, Zhang Z, et al: CD147, MMP-1, MMP-2 and MMP-9 protein expression as significant prognostic factors in human prostate cancer. Oncology 75: 230-236, 2008.

10. Peters JL, Sutton AJ, Jones DR, Abrams KR and Rushton L: Comparison of two methods to detect publication bias in meta-analysis. JAMA 295: 676-680, 2006.

11. Jia ZY, Ma M and Yu NN: Expression and significance of COX-2 and MMP-2 in prostate cancer. Shandong Med J 50: $52-53,2010$.

12. Ma Z: Expression of CD147 and MMP-2 in prostate cancer tissues and the correlation study of it. Tianjin: 9-12, 2014 (In Chinese).

13. Li DD: The expression and significance of MMP-2 and TIMP-2 in benign and malignant prostatic lesions. Tianjin: 9-11, 2013 (In Chinese)

14. Escaff S, Fernández JM, González LO, Suárez A, González-Reyes S, González JM and Vizoso FJ: Comparative study of stromal metalloproteases expression in patients with benign hyperplasia and prostate cancer. J Cancer Res Clin Oncol 137: 551-555, 2011.

15. Zhang XY, Hong BF, Chen GF, Lu YL and Zhong M: Significance of MMP2 and MMP9 expression in prostate cancer. Zhonghua Nan Ke Xue 11: 359-361, 364, 2005 (In Chinese)

16. Wu YD, Liu BQ, Zhang XP, et al: Detection osteopontin and matrix metalloproteinase-2 in prostate cancer. Zhengzhou Da Xue Xue Bao Yi Xue Ban 40: 654-656, 2005 (In Chinese).

17. Wu J and Zhang C: Expression of VEGF MMP-2 and MVD in prostatic cancer and its relation to clinic pathology. Mod Med Health 25: 3203-3204, 2009.

18. Center MM, Jemal A, Lortet-Tieulent J, Ward E, Ferlay J, Brawley $\mathrm{O}$ and Bray $\mathrm{F}$ : International variation in prostate cancer incidence and mortality rates. Eur Urol 61: 1079-1092, 2012.

19. Zhang L, Ji GY, Li XM, et al: The influence of mass screening for prostate cancer on the diagnostic status of the clinical prostate cancer. Chin J Urol 25: 103-105, 2004.

20. Amălinei C, Căruntu ID, Giuşcă SE and Bălan RA: Matrix metalloproteinases involvement in pathologic conditions. Rom J Morphol Embryol 51: 215-228, 2010.

21. Visse R and Nagase H: Matrix metalloproteinases and tissue inhibitors of metalloproteinases: Structure, function, and biochemistry. Circ Res 92: 827-839, 2003.

22. Deryugina EI and Quigley JP: Matrix metalloproteinases and tumor metastasis. Cancer Metastasis Rev 25: 9-34, 2006.

23. Liotta LA, Tryggvason K, Garbisa S, Hart I, Foltz CM and Shafie S: Metastatic potential correlates with enzymatic degradation of basement membrane collagen. Nature 284: 67-68, 1980.

24. Stetler-Stevenson WG: The role of matrix metalloproteinases in tumor invasion, metastasis, and angiogenesis. Surg Oncol Clin N Am 10: 383-392, 2001.

25. Stetler-Stevenson WG: Type IV collagenases in tumor invasion and metastasis. Cancer Metastasis Rev 9: 289-303, 1990.

26. Gohji K, Fujimoto N, Hara I, Fujii A, Gotoh A, Okada H, Arakawa S, Kitazawa S, Miyake H, Kamidono S, et al: Serum matrix metalloproteinase- 2 and its density in men with prostate cancer as a new predictor of disease extension. Int J Cancer 79: 96-101, 1998

27. Zhang L, Shi J, Feng J, Klocker H, Lee C and Zhang J: Type IV collagenase (matrix metalloproteinase-2 and -9) in prostate cancer. Prostate Cancer Prostatic Dis 7: 327-332, 2004.

28. Lovaas JD, Zhu L, Chiao CY, Byles V, Faller DV and Dai Y: SIRT1 enhances matrix metalloproteinase-2 expression and tumor cell invasion in prostate cancer cells. Prostate 73: 522-530, 2013. 